\title{
MICROSCOPIC PHYSICS IN THE SOLAR STANDARD MODEL
}

\author{
ILIDIO LOPES ${ }^{1}$ AND SYLVAINE TURCK-CHIEZE \\ Service d'Astrophysique, DAPNIA, CE Saclay, \\ 91191 Gif-sur-Yvette Cedex 01, France
}

ABSTRACT The microphysics of the solar standard model is revised. We discuss the contribution of composition, nuclear reaction rates, screening effect and opacity (OPAL project) to the theoretical predictions on neutrino flux and acoustic modes.

\section{INTRODUCTION}

The multiplication of the observed solar neutrino fluxes coupled with their sensitivity to the temperature $\left(\Phi(p p) \propto T_{C}^{-1.2}, \Phi\left({ }^{7} B e\right) \propto T_{C}^{8}\right.$ and $\left.\Phi\left({ }^{8} B\right) \propto T_{C}^{18}\right)$ and the high accuracy in the measurement of the five-minute oscillations (leading to an accurate sound speed, $c_{z}^{2} \propto T / \mu$ ) impose a revision of the nuclear and atomic physics strongly dependent of the temperature.

\section{MICROPHYSICS IMPROVEMENTS}

\section{Reaction Rates and Screening Factor}

We follow the reaction rates compiling by Caughlan and Fowler 1988 , except for $(p, p)$, $\left({ }^{3} \mathrm{He}_{1}{ }^{3} \mathrm{He}\right)$ and ${ }^{7} \mathrm{Be}(p, \gamma)$ (Turck-Chièze and Lopes 1992). The two calculations recently published to redetermine the $p+p$ reaction by Gould and Guessoum 1992 and Carlson et al 1991 present a difference of about $5 \%$ on the astrophysical factor $S(0)$. This difference has small consequences on the solar structure and on the gallium prediction, but leads to a $10 \%$ difference on the ${ }^{8} B$ neutrino flux. In the central region of the Sun, the atoms are, except for iron, completely stripped and the shielding effect of the free electrons reduces the coulomb potential and increases the thermonuclear reaction rates within the star by a factor $f=\exp \Lambda$, where $\Lambda$ is the ratio of the Coulomb interaction energy to the kinetic energy. If this hypothesis of weak screening (WS) $\left(\Lambda=E_{D} /(k T)<<1\right.$, Salpeter and Van Horn 1969), seems justified in the Sun for the main $p p$ reaction, $\Lambda$ may reach $0.2,0.4$ in a proton interaction with ${ }^{7} B e$ or ${ }^{14} \mathrm{~N}$, so the hypothesis of weak screening is questionable. Graboske et al 1973, considers an intermediate regime(IS) defined for $\Lambda>0.1$. Their general treatment in terms of chemical potentials was considered in our previous calculation (Turck-Chièze et al 1988) but the limitation of such approach comes from the negligence of nuclear and dynamical effects. As this effect concerns high $\mathrm{Z}$ ions, its main consequence is a slight modification of the branching ratio of the $p p$ chains and the CNO cycle (see table 1: model $c$ and d). It mainly affects the neutrinos connected to high $Z$ reactions, and pratically not the present structure of the Sun and consequently the acoustic frequencies. We consider in a recent work that this treatment underestimates the effect of screening and suggest a better adapted treatment which is compatible with the mean value of WS and IS (Dzitko et al 1992).

\footnotetext{
${ }^{1}$ Also, Centro de Astrofísica, Universidade do Porto, Rua do Campo Alegre, 823, 4100 Porto - PORTUGAL
} 


\begin{tabular}{|crrrrr|}
\hline Solar Model & model $a$ & model $b$ & model $c$ & model $d$ & model $\mathrm{e}$ \\
\hline \hline$T_{c}\left(10^{6} \mathrm{~K}\right)$ & 15.47 & 15.33 & 15.49 & 15.50 & 15.43 \\
$\rho_{c}\left(\mathrm{~g} / \mathrm{cm}^{3}\right)$ & 141.80 & 142.80 & 147.30 & 147.20 & 146.89 \\
$Y_{\text {initial }}$ & 0.2736 & 0.2664 & 0.2762 & 0.2760 & 0.2714 \\
$r_{B} C Z$ & 0.729 & 0.733 & 0.721 & 0.721 & 0.725 \\
$T_{B C Z}\left(10^{6} \mathrm{~K}\right)$ & 2.00 & 2.10 & 2.16 & 2.16 & 2.11 \\
\hline$\Phi(p p) / 10^{10}\left(\mathrm{~cm}^{-2} \mathrm{~s}^{-1}\right)$ & 6.05 & 6.08 & 6.02 & 5.99 & 6.01 \\
$\Phi(p e p) / 10^{8}\left(\mathrm{~cm}^{-2} \mathrm{~s}^{-1}\right)$ & 1.29 & 1.30 & 1.38 & 1.38 & 1.39 \\
$\Phi\left({ }^{7} \mathrm{Be}\right) / 10^{9}\left(\mathrm{~cm}^{-2} \mathrm{~s}^{-1}\right)$ & 4.16 & 4.08 & 4.42 & 4.62 & 4.42 \\
$\Phi\left({ }^{8} \mathrm{~B}\right) / 10^{6}\left(\mathrm{~cm}^{-2} \mathrm{~s}^{-1}\right)$ & 4.04 & 4.28 & 4.51 & 5.15 & 4.71 \\
$\Phi\left({ }^{13} \mathrm{~N}\right) / 10^{8}\left(\mathrm{~cm}^{-2} \mathrm{~s}^{-1}\right)$ & 3.20 & 3.40 & 3.49 & 3.99 & 4.00 \\
$\Phi\left({ }^{15} \mathrm{O}\right) / 10^{8}\left(\mathrm{~cm}^{-2} \mathrm{~s}^{-1}\right)$ & 2.62 & 2.82 & 2.91 & 3.45 & 3.39 \\
\hline chlorine detector $(\mathrm{SNU})$ & 5.88 & 5.22 & 6.51 & 7.30 & 6.78 \\
water detector $\left./ 10^{6}\right)$ & 4.04 & 3.51 & 4.59 & 5.15 & 4.71 \\
gallium detector(SNU) & 120 & 116 & 123 & 126 & 124 \\
\hline
\end{tabular}

TABLE I Different predictions of the present Sun : Solar models computed with pp reaction rate proposed by Gould and Guessoum and intermediate screening with LAOL tables for high (model a) and low iron (model b) composition. The other models are computed with pp reaction rate calculated by Carlson et al with OPAL tables and the low iron composition. Model $c$ and $d$ use respectively IS or WS for reactions involving nuclei with $Z>3$. Model $e$ (comparable to d) introduces the new $C N O$ composition.( $1 S N U=10^{-36}$ captures per second per target atom, ${ }^{(*)}$ in $\mathrm{cm}^{-2} \mathrm{~s}^{-1}$ )

\section{Initial composition and opacity}

The radiative energy transport of the low mass stars needs precise estimates of the photon interactions ( Thomson scattering, free-free processes, bound-free and boundbound processes) with the low and heavy elements. For a precise calculation, one needs a proper determination of the initial composition ( the heavy elements contribute to $90 \%$ at the basis of the convective zone). Solar models have been recomputed with the Los Alamos (LAOL) Opacity library for the high and low iron ccmposition adjusting the ratio $\mathrm{Z} / \mathrm{H}$ accordingly to the observed values (see table 1 : model $a$ and $b$ ). One notices a difference of $13 \%$ on the chlorine and water neutrino flux detection. In the acoustic mode frequency predictions, we have a degradation of the order of $2 \mu \mathrm{Hz}$ for modes $l \leq 40$ and $\nu \approx 4500 \mu \mathrm{Hz}$ which are trapped in the intermediate region ( 0.4 $0.7 \mathrm{R} \odot$ ). The new CNO composition recently proposed by Grevesse et al 1991 reduces the ratio $Z / X$ by $9 \%$ and produced an effect of the same order on neutrino detection (model $d$ and $e$ ), (Turck-Chièze and Lopes 1992).

The improvements realised by the Livermore group (Rogers and Iglesias 1992) using OPAL code concern the equation of state and also the treatment of the mixture with coupled equations for the full solar composition. In the case of the Sun, their results differ from the LAOL estimate by less than $5 \%$ in the center and $20 \%$ in the intermediate region. The first consequence is a better determination of the intermediate region, illustrated in figure 1 by the determination of the $p$ - modes prediction. The second consequence is an increase on the central temperature of the same order but with an opposite sign than the effect of improved composition. 

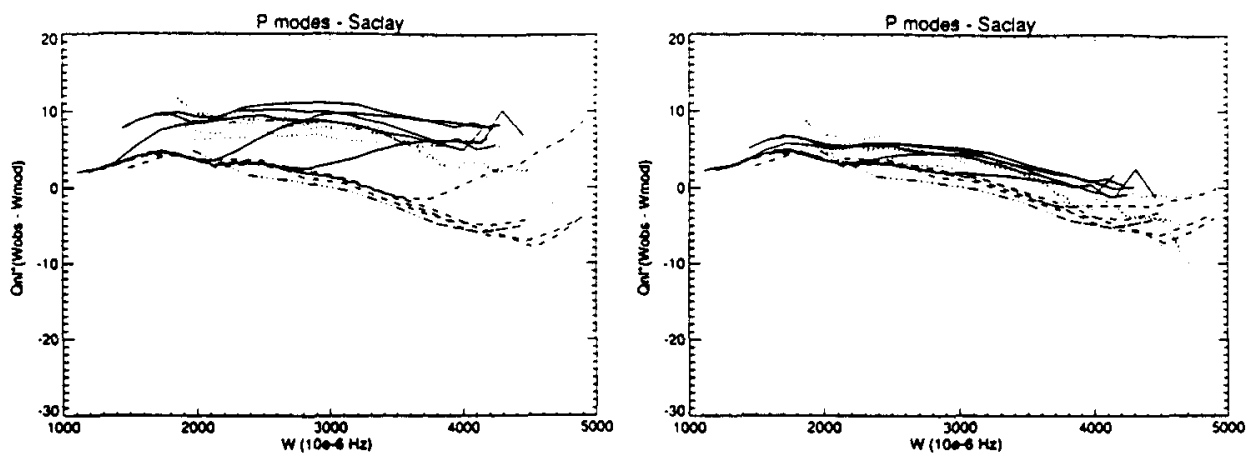

FIGURE I Scaled Frequency differences between observed frequencies of solar oscillations from the compilation by Libbrecht et al and theoretical frequencies of Solar Standard Model for selected values of $l$. Points correponding to a given value of $l$ have been connected, according to the following convention: $l=0-3$ (doted line), $l=4,5,10,20,30$ (continuous line), $l=40,50,70,100$ (dashed line), $l=150$ ( 9 doted line). The figure $1 \mathrm{a}$ belongs to the model $b$ and the figure $1 \mathrm{~b}$ belongs to the model $d$.

\section{DISCUSSION}

Improvements in the microscopic physics help us to better understand solar modelling and the determination of acoustic mode frequencies is a guide to qualify such model. The next step will be the revision or confirmation of the nuclear reaction rates and the treatment of the outerpart (molecular contribution to the opacity), before the introduction of the dynamical effects.

\section{ACKNOWLEDGMENTS}

We are particularly grateful to Jørgen Christensen-Dalsgaard who supplied us the pulsation code and to Werner Däppen who has introduced us to the helioseismological field. This work was partly financed by grant BD/749/90-RM from JNICT, Portugal.

\section{REFERENCES}

J. Carlson, D. O. Riska, R. Schiavilla, and R. B. Wiringa, Phys. Rev. C, $1991,44,619$. G. R. Caughlan and W. A. Fowler, 1988, Atomic Data and Nucl. Data Tab., 40, 283.

H. Dzitko, P. Delbourgo-Salvador, C. Lagrange, S. Turck-Chièze, 1992, International Symposium, June $22-25^{\text {th }}$, I.A.P, Paris.

H. C. Graboske, H. E. DeWitt, A.S. Grossman and M. S. Cooper, 1973, ApJ, $181,457$.

N. Grevesse, D. L. Lambert, A. J. Sauval, E. F. van Dishoeck, C. B. Farmer, and R. H. Norton, Astron. Astrophys.,1991, 242, 488.

R. J. Gould and N. Guessoum, 1992, ApJ, 359 , L67.

F. J. Rogers and C. A. Iglesias, 1992, $A p J S, 79,507$.

K.G. Libbrecht, M. F. Woodard and J. M. Kaufman, 1990, ApJS, 74, 1129.

E. E Salpeter and H. M. Van Horn, 1969, $A p J, 155,183$

S. Turck-Chièze, S. Cahen, M. Cassé and C. Doom, 1988, ApJ, $335,415$.

$\mathrm{S}$. Turck-Chièze and I. Lopes, 1992, $A p J$, to be published 